\title{
COMPORTAMIENTO DE HÍBRIDOS DE MAÍZ CON ALTA CALIDAD DE PROTEÍNA, POR SU BUEN RENDIMIENTO Y TOLERANCIA AL "ACHAPARRAMIENTO"1
}

\author{
Mauro Sierra-Macías ${ }^{2}$, Artemio Palafox-Caballero ${ }^{2}$, Enrique Noé Becerra-Leor ${ }^{2}$, Hugo Córdova-Orellana ${ }^{3}$, \\ Alejandro Espinosa Calderón ${ }^{4}$, Flavio A. Rodríguez-Montalvo ${ }^{2}$
}

\begin{abstract}
RESUMEN
Comportamiento de híbridos de maíz con alta calidad de proteína, por su buen rendimiento y tolerancia al "Achaparramiento". Con los objetivos de conocer el rendimiento y las características agronómicas de híbridos de maíz con alta calidad de proteína (QPM) e identificar los que mejor se adapten y presenten tolerancia a la enfermedad "Achaparramiento", durante el ciclo primavera verano 2005 se establecieron experimentos en las localidades de Cotaxtla, municipio de Medellín de Bravo, La Torrecilla, Municipio de Tlalixcoyan y Zapotal Número Uno, municipio de Ignacio de la Llave, en el estado de Veracruz. Los ensayos fueron sembrados bajo un diseño alpha látice $9 \times 2$ con 18 tratamientos y tres repeticiones, en parcelas de dos surcos de $5 \mathrm{~m}$ de largo separados a $80 \mathrm{~cm}$, con una densidad de 62,500 plantas/ha. Durante el desarrollo del cultivo se registraron las variables: rendimiento de grano, porcentaje de plantas y mazorcas dañadas por achaparramiento, severidad del daño, altura de planta y de mazorca, días a floración masculina y femenina, aspecto y sanidad de planta y mazorca, porcentaje de plantas acamadas, porcentaje de mazorcas con mala cobertura y porcentaje de mazorcas podridas. De los análisis de varianza combinados para: Rendimiento de grano, porcentaje de daño y severidad por achaparramiento en planta y porcentaje de mazorcas con achaparramiento, se encontró diferencia altamente significativa para híbridos $(\mathrm{H})$ y para localidades (L). La interacción HxL, excepto para rendimiento de grano, en el resto de las variables fue significativa. Los híbridos QPM que registraron el mayor rendimiento, tolerancia al achaparramiento y mejores características agronómicas fueron: (CML264QxCML150)xCML491, (CML264Qx CML273Q)xCML491,(CML144xCML159)xCLQ-RCWQ50 y (CML492x CML150)xCLQ-RCWQ50.
\end{abstract}

Palabras clave. Dalbulus maydis, Spiroplasma kunkelii, Phytoplasma, enanismo arbustivo del maíz, trópico húmedo, híbridos tolerantes.

\begin{abstract}
Quality protein maize hybrids with high yield and tolerants to corn stunt disease. The objectives of this research were to determine the yield and agronomic characteristics of Quality Protein Maize (QPM) hybrids, and to identify those well adapted and tolerant to corn stunt disease. Thus, during the spring summer season in 2005, there were carried out three experiments of QPM hybrids at Medellín de Bravo, Tlalixcoyan and Ignacio de la Llave in Veracruz, México. The QPM hybrid trials were planted under a $9 \times 2$ alpha lattice design with 18 entries and three replications, in plots of two rows $5 \mathrm{~m}$ long and $80 \mathrm{~cm}$ wide, and 62,500 plants/ha. Data were taken in each two-row plots, included grain yield, percentage of damaged plants and ears for corn stunt, severity of damage, plant and ear height, days to pollen shed and silk emergence, plant and ear aspect, percentage of lodging, percentage of ears with bad husk cover and ear rot. From the combined analysis, the variation for grain yield, percentage of damaged plants and ears for corn stunt and severity was highly significant differences for hybrids $(\mathrm{H})$ and locations (L). The interaction HxL was significant for all traits, except for grain yield. The QPM hybrids that registered the best yield, agronomic characteristics and tolerance to corn stunt were: (CML264Qx CML150)xCML491, (CML264QxCML 273Q)xCML491, (CML144xCML159)xCLQ-RCWQ50, (CML492 x CML150)x CLQ-RCWQ50.
\end{abstract}

Key words: Dalbulus maydis, Spiroplasma kunkelii, Phytoplasm, maize bushy stunt, humid tropic, tolerant hybrids.

1 Recibido: 10 de mayo, 2006. Aceptado: 5 de marzo, 2007. Parte del proyecto "Combatiendo el hambre oculta en América Latina: Cultivos Biofortificados con vitamina A, minerales esenciales y proteína de calidad mejorados". Presentado en la LII Reunión del PCCMCA, Nicaragua. 2006.

2 Instituto Nacional de Investigaciones Forestales, Agrícolas y Pecuarias. Centro de Investigación Regional Golfo Centro. Campo Experimental Cotaxtla. Apdo. Postal 429, Km. 34 Carr. Veracruz-Córdoba, Veracruz, Veracruz, México. CP 91700. Tel. 01 (229) 934-83-54. Correo electrónico: mauro_s55@hotmail.com, palafox012@hotmail.com, becenle@yahoo.com.mx,rodríguez.flavio@inifap.gob.mx

3 Centro Internacional de Mejoramiento de Maíz y Trigo. Km 45 carretera México-Veracruz.El Batán Texcoco, Edo. De México. C.P. 56130 Correo electrónico: h.cordova@cgiar.org

4 Instituto Nacional de Investigaciones Forestales, Agrícolas y Pecuarias. Campo Experimental Valle de México. Correo electrónico: espionale@ yahoo.com.mx 


\section{INTRODUCCIÓN}

En México el cultivo de maíz (Zea mays L.) es el más importante por su superficie sembrada, valor de la producción, por ser el alimento principal de la población y por ocupar el 20 por ciento de la población económicamente activa.

En 2002 se sembraron en México 6,48 millones de hectáreas con este cultivo, con un rendimiento de 2,32 t/ha (SAGARPA 2002). En el área tropical se siembran 2,5 millones de hectáreas, de las cuales un millón están comprendidas en provincias agronómicas de buena, y muy buena productividad, y donde es factible el uso de semilla mejorada de híbridos y variedades sintéticas (Sierra et al. 2001).

En México existen 31 millones de personas con desnutrición, de los cuales, 18 millones padecen desnutrición severa, se trata de diez millones de indígenas y el resto es población urbana de bajos ingresos. En este marco, el maíz es fundamental en la alimentación de los mexicanos, ya que se calcula un consumo aparente de 209,8 kilogramos por persona (Morris y López 2000).

El maíz con alta calidad de proteína deriva del aprovechamiento del gen mutante opaco 0202 , el cual en su versión homocigótica recesiva, se expresa con mayor contenido de lisina y triptófano, aminoácidos esenciales en la alimentación (Mertz 1994). Vasal et al. (1994) concentraron genes modificadores del endospermo para lograr la textura cristalina o dentada en el grano, que expresa el gene 0202 , obteniendo con ello líneas, híbridos y variedades con la textura del maíz común, con características aptas para la industria, para consumo tradicional.

La enfermedad del "achaparramiento" ha causado pérdidas en varios países de América, entre ellos: Estados Unidos, México, Honduras, El Salvador, República Dominicana, Haití, Nicaragua, Panamá y Argentina (Henríquez y Jeffers 1997; Gordon et al. 1997) y ha sido un factor limitante en zonas tropicales y subtropicales. En Centroamérica y el Caribe, la enfermedad alcanza niveles críticos de incidencia, principalmente en regiones donde los agricultores siembran variedades criollas con bajo nivel tecnológico y donde las condiciones climatológicas son favorables para el desarrollo del vector, como son: escasez de lluvia, altas temperaturas y baja humedad relativa (Urbina 1997; Ibarra-Aparicio et al. 2005). En México se han reportado daños en la península de Yucatán y en la región costera del Golfo, principalmente en siembras tardías, registrándose daños mayores al $25 \%$ en siembras comerciales (Cano et al. 2000; Sierra et al. 2004 b).

La enfermedad es provocada al interactuar sobre la planta tres patógenos: el virus del rayado fino del maíz MRFV (Marafavirus), el espiroplasma del maíz CSS (Spiroplasma kunkelii) y el Phytoplasma del enanismo arbustivo del maíz MBSM. Los insectos vectores que lo diseminan son la chicharrita Dalbulus maydis (DeLong y Wolcott) y D. Elimatus Ball. (Bajet y Renfro 1989; Henríquez y Jeffers 1997). De León (1984), Henríquez y Jeffers (1997) citan que el MRFV induce la formación de finas manchitas cloróticas en las hojas que se consideran características de la enfermedad. Las primeras manchitas ocurren en las hojas del cogollo y se unen progresivamente para formar manchas alargadas en toda la hoja, produciendo el rayado. En genotipos susceptibles puede llegar a reducir el tamaño de la planta y confundirse con infección de CSS o MBSM. Los primeros síntomas ocurren de ocho a 14 días después de la inoculación, pero en invernaderos y con temperatura controlada se han observado síntomas de los cinco a seis días en maíz Tuxpeño Sequía (Henríquez y Jeffers 1997). El agente causal del CSS es un micoplasma helicoidal o Spiroplasma que mide de 0,15 a $0,2 \mathrm{~nm}$ de diámetro y cerca de 2 a $15 \mathrm{~nm}$ de largo. Los síntomas se observan como bandas anchas sobre la base de las hojas jóvenes, las plantas sufren enanismo debido al acortamiento de entrenudos, las yemas axilares desarrollan mazorcas estériles (Shurtleff 1980; De León 1984).

El Centro Internacional de Mejoramiento de Maíz y Trigo (CIMMYT) y el Programa Regional de Maíz en Centroamérica (PRM) han desarrollado híbridos tropicales de cruza simple y trilineal utilizando germoplasma resistente al complejo del achaparramiento (Jeffers et al. 2004). Al evaluarse 10 híbridos, con y sin infestación de D. maydis en Cotaxtla, Veracruz, se determinó que el híbrido $\mathrm{H}-371 \mathrm{C}$ rindió 4,66 t/ha y el híbrido H-513 registró un rendimiento de 2,27 t/ha, la tolerancia se debió principalmente a que los genotipos 
registraron menor porcentaje de plantas y mazorcas con sintomatología del complejo del achaparramiento (Sierra et al. 2004 b). El INIFAP generó el híbrido H520, el cual es un híbrido trilineal que usa como progenitor hembra el híbrido H-513 y es tolerante a la enfermedad del achaparramiento (Sierra et al. 2004 a).

Varios autores, han encontrado correlación negativa entre el rendimiento de grano y el síntoma de la enfermedad del achaparramiento en planta y mazorca, lo que sugiere que la enfermedad tiene un efecto directo sobre el rendimiento. Además han encontrado correlación entre los porcentajes de plantas y de mazorcas achaparradas (Gordon et al. 1997; Obando et al. 1997).

Los objetivos de este trabajo fueron: conocer el rendimiento y características agronómicas de híbridos de maíz con alta calidad de proteína; identificar aquellos con tolerancia a la enfermedad del "achaparramiento", y conocer el efecto de la enfermedad del achaparramiento en el rendimiento de grano de los híbridos evaluados.

\section{MATERIALES Y MÉTODOS}

\section{Localización}

Durante el ciclo primavera verano 2005, se estableció un ensayo uniforme de híbridos de maíz QPM provenientes del CIMMYT en las localidades de Cotaxtla, Municipio de Medellín de Bravo, La Torrecilla, Municipio de Tlalixcoyan, y El Zapotal Número Uno, Municipio de Ignacio de la Llave en el estado de Veracruz, México, que de acuerdo con la clasificación climática de Köppen modificada por García (1981), pertenecen a los climas $\mathrm{Aw}_{1}, \mathrm{Aw}_{2}$ (w)(e)g, $\mathrm{Aw}_{2}{ }_{2}(\mathrm{w})(\mathrm{i}$ ') para las tres localidades respectivamente y que corresponden a los climas cálido subhúmedo con lluvias en verano. $\mathrm{Aw}_{1}$ con un cociente precipitación: temperatura entre 43,2 y 55,3 y el caso de $\mathrm{Aw}_{2}$ mayor de 55,3 y es el más húmedo de los subhúmedos.

\section{Germoplasma utilizado}

Se incluyeron 16 híbridos trilineales experimentales con alta calidad de proteína y los híbridos normales H-59 RE y H-520 utilizados como testigo.

\section{Descripción de los experimentos}

Los experimentos fueron establecidos el 5 de julio para la localidad de Cotaxtla; el 11 y 12 de julio del 2005, para las localidades de La Torrecilla y Número Uno, respectivamente. Se sembró bajo un diseño alpha látice $9 \times 2$ con 18 tratamientos y tres repeticiones en parcelas de dos surcos de $5 \mathrm{~m}$ de largo separados a $80 \mathrm{~cm}$, a la siembra se depositaron dos semillas cada $20 \mathrm{~cm}$ y se dejó una planta por sitio de siembra antes del atierre es decir, se tuvo una densidad de 62.500 plantas/ha. El control de las malezas fue a base de Atrazina en dosis de $3 \mathrm{~kg} / \mathrm{ha}$. Se fertilizó con la fórmula recomendada para maíces normales 161-46-00 aplicando $100 \mathrm{~kg}$ de urea más $100 \mathrm{~kg}$ de Super fosfato de calcio triple (SPCT) durante los primeros 10 días a partir de la siembra y el resto del nitrógeno al momento del atierre.

\section{Variables y registro de datos}

Durante el desarrollo del cultivo se registraron las variables agronómicas: rendimiento de grano, porcentaje de plantas con daño de achaparramiento, severidad del daño por achaparramiento con escala de 1 a 9 donde, 1 es el menor daño y 9 representa el daño mayor (tomando en cuenta la sintomatología de la enfermedad coloración amarillo-rojizo de las hojas en los tercios de la planta, reducción de la altura y proliferación de mazorcas). La variable porcentaje de achaparramiento en planta se registró durante el período de llenado de grano previo a la dobla (21 de septiembre), en Ignacio de la Llave, y 7 de octubre en La Torrecilla. La variable severidad de daño por achaparramiento en planta se registró el 30 de septiembre en las tres localidades. En el porcentaje de mazorcas con daño de achaparramiento fue registrado al momento de la cosecha y se consideraron mazorcas pequeñas, chupadas y sin grano, altura de planta y de mazorca, días a floración masculina y femenina, aspecto y sanidad de planta y de mazorca con escala de 1 a 5 donde 1 es lo mejor y 5 es lo peor, porcentaje de plantas acamadas, porcentaje de mazorcas con mala cobertura, y porcentaje de mazorcas podridas.

Métodos estadísticos. Se hicieron análisis de varianza individuales para las variables en estudio y análisis combinado para rendimiento, porcentaje de 
plantas y severidad por achaparramiento en planta y porcentaje de daño por achaparramiento en mazorca. Los datos registrados en porcentaje fueron transformados a grados angulares (bliss), para proceder al análisis de varianza. Se hicieron correlaciones fenotípicas entre las variables: rendimiento de grano con porcentaje de daño por achaparramiento en planta y mazorca, severidad, porcentaje de daño en planta con porcentaje de daño en mazorca. La separación de medias se hizo mediante la prueba DMS.

\section{RESULTADOS Y DISCUSIÓN}

De los análisis de varianza combinado para las localidades de Cotaxtla, Tlalixcoyan e Ignacio de la Llave, Veracruz, para las variables rendimiento de grano, porcentaje de daño y severidad por achaparramiento en planta y porcentaje de daño en mazorca, se encontró significancia estadística al 0,01 de probabilidad para híbridos $(\mathrm{H})$ y localidades (L) en las cuatro variables. Para la interacción $\mathrm{HxL}$, excepto para rendimiento de grano, en el resto de las variables se encontró significancia estadística. Sin embargo, en las cuatro variables fue más importante la varianza debida al factor localidades, lo que significa que la mayor diferencia en el comportamiento de los híbridos fue debida al factor localidades. Los coeficientes de variación registrados fueron $12,58 \%$ para rendimiento de grano, $17,01 \%$ para porcentaje de daño por achaparramiento en planta, $29,56 \%$ para severidad de daño y 25,09\% para porcentaje de mazorcas dañadas por achaparramiento; valores relativamente bajos, que sugieren que la conducción de los experimentos y los resultados obtenidos son confiables (Cuadro 1).

\section{Rendimiento de grano}

Se encontró un grupo de cinco híbridos que fueron significativamente superiores a través de las tres localidades de evaluación al 0,05 de probabilidad, tales híbridos fueron: (CML492 x CML150) x CLQRCWQ50, (CML264Q x CML273Q) x CML491, (CML-264QxCML150) x CML-491, (CML264Q x CML-491) x CLQ-RCWQ10, (CML-144 x CML-159) x CLQ-RCWQ50, los cuales superaron numéricamente a los testigos H-59 RE y H-520 de un 11 a $22 \%$ (Cuadro 2). Las ventajas agronómicas de rendimiento y
Cuadro 1. Cuadrados medios y significancia en el análisis de varianza combinado para rendimiento de grano, porcentaje de daño por achaparramiento en planta y mazorca y severidad. Híbridos QPM. Veracruz, México. 2005 B.

\begin{tabular}{lllll}
\hline $\begin{array}{c}\text { Fuente de } \\
\text { variación }\end{array}$ & RG & PPA & SDA & PDAM \\
\hline Híbridos (H) & $5,0^{* *}$ & $5,1^{* *}$ & $6,9 * *$ & $8,1^{* *}$ \\
Localidades (L) & $96,8^{* *}$ & $116,4^{* *}$ & $8,0^{* *}$ & $85,6^{* *}$ \\
Interacción HxL & $0,58 \mathrm{NS}$ & $1,23 * *$ & $1,37 *$ & $1,8^{*}$ \\
Error & 0,42 & 0,55 & 0,75 & 1,17 \\
CV $(\%)$ & 12,58 & 17,01 & 29,16 & 25,09 \\
\hline
\end{tabular}

$\mathrm{RG}=$ Rendimiento de grano; PPA= Porcentaje de plantas con achaparramiento; SDA = Severidad de daño por achaparramiento; PDAM = Porcentaje de daño por achaparramiento en mazorca; $\mathrm{CV}=$ Coeficiente de variación;

* =Significancia de los tratamientos al 0,05 de probabilidad;

$* *=$ Significancia de los tratamientos al 0,01 de probabilidad

características agronómicas de estos híbridos, representa una alternativa de producción para los agricultores maiceros en el estado de Veracruz, con la ventaja adicional de mayor contenido de lisina y triptófano con relación a los maíces normales como lo mencionaron Mertz (1994) y Vasal et al. (1994) y ayudaría a mejorar la nutrición en los consumidores Morris y López (2000). Las localidades de Tlalixcoyan e Ignacio de la Llave, registraron los rendimientos medios más bajos, debido a la mayor presencia de la enfermedad del "achaparramiento".

\section{Daño por "achaparramiento"}

Los síntomas principales observados fueron la coloración rojiza y la proliferación de jilotes que corresponden al patógeno Phytoplasma del enanismo arbustivo del maíz (MBSM); en menor proporción se presentaron síntomas de bandas blancas y amarillas que corresponde a la presencia del patógeno Spiroplasma del maíz (CSS); no se observaron los síntomas de rayado fino (MRFV) Marafavirus, de acuerdo con los síntomas descritos por Shurtleff (1980); De León (1984); Bajet y Renfro (1989); y Henríquez y Jeffers (1997). Los daños de la enfermedad del achaparramiento en mazorca se manifestó por la expresión de mazorcas chupadas, de bajo peso y limitada formación de grano (Shurtleff 1980; De León 1984). 
Cuadro 2. Rendimiento de grano de híbridos de maíz con alta calidad de proteína. Veracruz, México. 2005 B.

\begin{tabular}{rlrccc}
\hline Trat & \multicolumn{1}{c}{ Genealogía } & COT & TLALIX & IG LLAVE & PROM \\
\hline \multirow{2}{*}{8} & (CML-492xCML-150)xCLQ-RCWQ50 & 7,82 & 5,46 & 5,6 & $6,29^{*}$ \\
10 & (CML-264QxCML-273Q)xCML-491 & 7,49 & 5,11 & 5,13 & $5,91^{*}$ \\
6 & (CML-264QxCML-150)xCML-491 & 7,18 & 4,71 & 5,74 & $5,88^{*}$ \\
13 & (CML-264QxCML-491)xCLQ-RCWQ10 & 7,32 & 5,32 & 4,94 & $5,86^{*}$ \\
7 & (CML-144xCML-159)xCLQ-RCWQ50 & 7,47 & 5,29 & 4,32 & $5,69^{*}$ \\
3 & (CLQ-RCWQ10xCLQ-6315)xCML-491 & 6,96 & 4,66 & 5,2 & $5,61^{* *}$ \\
9 & (CML-492xCML-146)xCLQ-RCWQ50 & 7,25 & 4,89 & 4,32 & $5,49^{*} *$ \\
17 & H-59 RE & 6,45 & 5,61 & 4,08 & 5,38 \\
12 & (CLQ-RCWQ10xCLQ-6315)xCML-264Q & 7,10 & 4,19 & 4,11 & 5,13 \\
18 & H-520 & 7,00 & 4,67 & 3,71 & 5,13 \\
4 & (CML-141xCML-142)xCML-491 & 5,72 & 4,85 & 4,64 & 5,07 \\
5 & (CML-264QxCML-159)xCML-491 & 6,37 & 4,62 & 4,1 & 5,03 \\
11 & (CL-RCWQ13xCLQ-6316)xCML-264Q & 6,73 & 3,68 & 4,39 & 4,93 \\
14 & (CML-264QxCML-491)xCLQ-6315 & 6,50 & 4,41 & 3,64 & 4,85 \\
2 & (CML-492xCML-150)xCML-491 & 6,28 & 4,51 & 3,68 & 4,82 \\
15 & (CML-264QxCML491)xCLQ-6316 & 6,49 & 3,74 & 3,89 & 4,71 \\
16 & (CML-144xCML-159)xCML-176 RE & 5,66 & 2,98 & 2,13 & 3,59 \\
1 & (CML-264QxCML-492)xCML-491 & 4,56 & 2,62 & 3,09 & 3,40 \\
\hline & PROMEDIO & 6,69 & 4,52 & 4,26 & 5,15 \\
& CV (\%) & 11,42 & 10,84 & 13,71 & 12,58 \\
& CME & 0,58 & 0,24 & 0,45 & 0,42 \\
& SIG. F & 1 & 1 & 1 & 1 \\
\hline & DMS 0,05 & 1,26 & 0,81 & 1,11 & 0,6 \\
& DMS 0,01 & 1,69 & 1,09 & 1,49 & 0,8 \\
\hline
\end{tabular}

* Significancia de los tratamientos al 0,05 de probabilidad

** Significancia de los tratamientos al 0,01 de probabilidad; CV $=\%$ Coeficiente de variación COT = Cotaxtla, TLALIX = Tlalixcoyan, IG LLAVE = Ignacio de la Llave; PROM = Promedio. $\mathrm{CME}=$ Cuadrado medio del error; Sig. F = Significancia de F.

Por lo que se refiere al porcentaje de daño por achaparramiento en planta, a través de las localidades de Cotaxtla, Tlalixcoyan e Ignacio de la Llave, Veracruz (Cuadro 3), se registraron valores de 10,37; 39,79 y $17,84 \%$ para cada localidad respectivamente y un promedio de 21,67\%. En relación con los híbridos, se encontró un grupo de seis híbridos que tuvieron el menor daño y fueron sobresalientes al 0,01 de probabilidad, tales fueron: (CML264QxCML150)xCML491, CML264QxCML273Q)xCML491, (CML144x CML159)xCLQRCWQ50, H-59 RE, H-520 y (CML492xCML150)xCLQ-RCWQ50.

En cuanto a la severidad de daño por achaparramiento, un grupo de siete híbridos tuvo significativamente menor al 0,01 de probabilidad, tales fueron: (CML492xCML150)xCLQ-RCWQ50, (CML264Qx CML273Q)xCML491, (CML264QxCML150) x
CML491, H-520, (CML264QxCML159)xCML491, (CL-RCWQ13xCLQ6315)xCML264Q, H-59RE y (CML144xCML159)xCLQ-RCWQ50, los cuales registraron valores menores a 2,6 , es decir presentan severidad baja.

En relación con el porcentaje de mazorcas con daño por "achaparramiento" la localidad de Cotaxtla tuvo un porcentaje de daño de 8,53\%, Tlalixcoyan tuvo $27,57 \%$ e Ignacio de la Llave tuvo 29,39\%, y a través de las tres localidades un promedio general de $21,83 \%$. Con relación a los híbridos, seis de ellos fueron sobresalientes al 0,01 de probabilidad entre ellos: (CML264QxCML273Q)xCML491, CML264Qx CML150)xCML491, (CML264QxCML159)x CML491, (CML492xCML150)xCLQ-RCWQ50, CML264QxCML159)xCML491 y (CML264Qx CML150)xCLQ-RCWQ50. 
Cuadro 3. Porcentaje de daño y severidad por "achaparramiento" en planta y porcentaje de daño en mazorca de híbridos de maíz con alta calidad de proteína. Veracruz, México. 2005B.

\begin{tabular}{|c|c|c|c|c|c|c|c|c|c|c|c|c|c|}
\hline \multirow[t]{2}{*}{ Trat } & \multirow[t]{2}{*}{ Genealogía } & \multicolumn{3}{|c|}{ Cotaxtla } & \multicolumn{3}{|c|}{ Tlalixcoyan } & \multicolumn{3}{|c|}{ Ignacio de la Llave } & \multicolumn{3}{|c|}{ Promedio } \\
\hline & & PPA & $\mathbf{S}$ & PMA & PPA & $\mathbf{S}$ & PMA & PPA & $\mathbf{S}$ & PMA & $\overline{\text { PPA }}$ & $\mathbf{S}$ & PMA \\
\hline \multicolumn{14}{|c|}{6 (CML264QxCML150)x } \\
\hline & CML491 & 3,54 & 1,33 & 1,96 & 26,06 & 2,67 & 20,41 & 2,54 & 1,67 & 16,00 & $10,71 *$ & $1,89^{*}$ & $12,79 *$ \\
\hline 10 & CML264QxCML273Q) xCML491 & 6,95 & 1,33 & 0,74 & 20,3 & 2,00 & 6,06 & 5,73 & 1,67 & 15,29 & $10,99 *$ & $1,67 *$ & $7,36^{*}$ \\
\hline \multirow{2}{*}{7} & (CML144xCML159) & & & & & & & & & & & & \\
\hline & xCLQ-RCWQ50 & 10,19 & 3,00 & 14,29 & 22 & 2,00 & 39,59 & 9,01 & 2,67 & 29,57 & $13,8 * *$ & $2,56^{* *}$ & 11,16 \\
\hline 17 & H-59 RE & 12,79 & 2,33 & 16,75 & 22,6 & 3,00 & 18,00 & 9,32 & 2,33 & 28,66 & $14,9^{* *}$ & $2,55^{* *}$ & 21,14 \\
\hline 18 & H-520 & 5,07 & 1,33 & 1,42 & 25,7 & 2,33 & 15,66 & 15,69 & 3,00 & 37,72 & $15,5^{* *}$ & $2,22 *$ & 18,27 \\
\hline \multirow{2}{*}{8} & (CML492xCML150) & & & & & & & & & & & & \\
\hline & xCLQ-RCWQ50 & 5,94 & 1,33 & 7,02 & 33,11 & 2,00 & 12,02 & 9,28 & 1,33 & 14,45 & $16,1 * *$ & $1,55^{*}$ & $19,33^{*}$ \\
\hline \multirow[t]{2}{*}{5} & (CML264QxCML159) & & & & & & & & & & & & \\
\hline & xCML491 & 6,87 & 1,33 & 4,79 & 29,65 & 2,33 & 10,75 & 21,8 & 3,33 & 12,91 & 19,44 & $2,33^{*}$ & $9,48^{*}$ \\
\hline \multirow[t]{2}{*}{13} & (CML264QxCML491) & & & & & & & & & & & & \\
\hline & xCLQ-RCWQ10 & 9,93 & 2,67 & 5,39 & 30,5 & 2,67 & 20,14 & 21,93 & 4,33 & 18,93 & 20,79 & 3,22 & $14,8^{* *}$ \\
\hline \multirow[t]{2}{*}{12} & (CLQ-RCWQ10X & & & & & & & & & & & & \\
\hline & CLQ-6315)CML264Q & 12,12 & 3,33 & 8,37 & 40,02 & 2,67 & 36,91 & 13,69 & 3,33 & 25,61 & 21,94 & 3,11 & 23,63 \\
\hline \multirow[t]{2}{*}{11} & (CLRCWQ13XCLQ6316) x & & & & & & & & & & & & \\
\hline & CML264Q & 7,05 & 2,33 & 8,55 & 41,71 & 2,00 & 31,68 & 17,63 & 3,33 & 47,86 & 22,13 & $2,55^{* *}$ & 29,36 \\
\hline \multirow{2}{*}{3} & (CLQ-RCWQ10 XCLQ-6315)x & & & & & & & & & & & & \\
\hline & CML491 & 12,00 & 4,33 & 8,11 & 53,14 & 4,00 & 34,99 & 13,20 & 3,67 & 26,29 & 26,11 & 4,00 & 23,13 \\
\hline \multirow{2}{*}{9} & (CML492xCML146) & & & & & & & & & & & & \\
\hline & xCLQ-RCWQ50 & 14,76 & 3,00 & 7,92 & 43,83 & 2,67 & 22,29 & 19,99 & 4,33 & 27,79 & 26,19 & 3,33 & 19,33 \\
\hline 2 & (CML492xCML150)CML491 & 15,2 & 3,33 & 19,00 & 38,3 & 2,67 & 39,01 & 25,8 & 3,67 & 21,72 & 26,43 & 3,22 & 26,58 \\
\hline 4 & (CML141xCML142)CML491 & 12,92 & 3,00 & 8,18 & 46,1 & 3,67 & 39,64 & 20,64 & 4,00 & 35,5 & 26,55 & 3,56 & 27,78 \\
\hline \multirow{2}{*}{15} & (CML264QxCML491) & & & & & & & & & & & & \\
\hline & xCLQ-6316 & 13,09 & 3,33 & 10,56 & 49,15 & 3,33 & 40,22 & 19,67 & 3,33 & 42,82 & 27,30 & 3,33 & 31,20 \\
\hline 1 & (CML264QxCML492)xCML491 & 10,53 & 3,67 & 6,59 & 54,24 & 3,33 & 11,43 & 21,3 & 3,33 & 30,16 & 28,69 & 3,44 & $16,1 * *$ \\
\hline 16 & (CML144xCML159)xCML176 RE & 11,33 & 4,33 & 12,80 & 36,55 & 3,33 & 58,56 & 45,55 & 7,00 & 39,44 & 31,14 & 4,89 & 36,93 \\
\hline \multirow[t]{7}{*}{14} & (CML264QxCML491)xCLQ-6315 & 16,41 & 3,00 & 11,14 & 49,28 & 4,00 & 38,90 & 28,44 & 5,00 & 58,33 & 31,38 & 4,00 & 36,12 \\
\hline & PROMEDIO & 10,37 & 2,68 & 8,53 & 39,79 & 2,82 & 27,57 & 17,84 & 3,41 & 29,39 & 21,67 & 2,97 & 21,83 \\
\hline & $\mathrm{CV}(\%)$ & 46,99 & 18,8 & 33,96 & 23,08 & 18,1 & 16,64 & 40,72 & 27,9 & 26,64 & 17,01 & 29,16 & 25,09 \\
\hline & SIG. F. & 0 & 1 & 5 & 1 & 1 & 5 & 1 & 1 & 0 & 1 & 1 & 1 \\
\hline & CME & 23,75 & 1,08 & 0,946 & 72,11 & 0,26 & 253,86 & 52,79 & 0,9 & 1,88 & 49,55 & 0,75 & 1,17 \\
\hline & DMS 0,05 & 8,02 & 1,72 & 9,06 & 14,07 & 0,85 & 26,41 & 12,04 & 1,57 & 24,96 & 6,57 & 0,81 & 12,18 \\
\hline & DMS 0,01 & 10,82 & 2,31 & 12,14 & 18,86 & 1,13 & 35,38 & 16,14 & 2,11 & 45,58 & 8,69 & 1,07 & 16,11 \\
\hline
\end{tabular}

$\mathrm{B}=$ Ciclo primavera verano

* Significancia de los tratamientos al 0,05 de probabilidad;

** Significancia de los tratamientos al 0,01 de probabilidad;

Trat. $=$ Tratamientos

PPA = Porcentaje de plantas con achaparramiento, $\mathrm{S}=$ Severidad del daño, PMA= Porcentaje de mazorcas con achaparramiento.

$\mathrm{CME}=$ Cuadrado medio del error. SIG. F = Significancia en la prueba de F

Los híbridos que registraron tolerancia a la enfermedad del achaparramiento fueron: (CML264Qx CML150)xCML491, CML264Qx CML273Q)x CML491, (CML144xCML159)x CLQ-RCWQ50, (CML492xCML150)xCLQ-RCWQ50, y (CML264Qx CML159)xCML491.

En general, los genotipos que fueron tolerantes a la enfermedad del achaparramiento fueron también los que registraron los rendimientos más altos, lo que significa que estos híbridos de maíz con alta calidad de proteína representan una alternativa en la producción de maíz en el estado de Veracruz.

Estos resultados sugieren que existe germoplasma resistente a la enfermedad, de acuerdo con Jeffers et al. (2004); Sierra et al. (2004a); Sierra et al. (2004b). Estos híbridos representan una alternativa para la producción de maíz en Veracruz y mejorar la dieta de los consumidores (Morris y López 2000). 
Por lo que se refiere a localidades, el Cuadro 4 muestra que para la localidad de Cotaxtla se registró el rendimiento de grano más alto $(6,69$ t/ha), en la variable porcentaje de daño en planta por achaparramiento, en Ignacio de la Llave se registró el valor promedio más alto 47,45 \%**, seguido de La Torrecilla, Municipio de Tlalixcoyan con $21,44 \%$ y el valor más bajo fue para Cotaxtla 6,57 \%; Así también, en la variable severidad fueron las localidades de Ignacio de la Llave y La Torrecilla, Veracruz, los que tuvieron los valores más altos $(5,4$ y 5,2 para cada localidad respectivamente). Particularmente, Tlalixcoyan e Ignacio de la Llave son áreas donde la siembra de maíz para la producción de elote y la siembra en fechas escalonadas no permite romper con el ciclo del vector, la chicharrita $D$. maydis. Así también, las condiciones climatológicas son propicias para su desarrollo y transmisión de la enfermedad. (Ibarra-Aparicio et al. 2005; Urbina 1997; Cano et al. 2000; Sierra et al. 2004b).

Cuadro 4. Significancia para localidades en el análisis combinado para las variables rendimiento de grano, porcentaje de daño por achaparamiento en planta, severidad y porcentaje de mazorcas con daño. Veracruz, México. 2005 B.

\begin{tabular}{llllc}
\hline Localidad & RG & PPA & S & PMA \\
\hline \multicolumn{2}{l}{ Ignacio de la Llave 4,26} & 17,84 & $3,41^{* *}$ & $29,39 *$ \\
La Torrecilla & 4,52 & $39,79 * *$ & 2,82 & $27,57 *$ \\
Cotaxtla & $6,69 * *$ & 10,37 & 2,68 & 8,53 \\
\hline
\end{tabular}

$\mathrm{B}=$ Ciclo primavera verano

$\mathrm{RG}=$ Rendimiento de grano

Ig. De la Llave = Ignacio de la Llave

PPA $=$ Porcentaje de plantas con achaparramiento, $\mathrm{S}=$ Severidad del daño, PMA= Porcentaje de mazorcas con achaparramiento.

* Significancia para localidades al 0,05 de probabilidad.

** Significancia para localidades al 0,01 de probabilidad.

\section{Características agronómicas}

Los híbridos sobresalientes en rendimiento y tolerancia a la enfermedad del "achaparramiento", registraron además características agronómicas deseables como son: altura de planta y mazorca intermedia baja, ciclo biológico intermedio (55 a 60 días a floración), buen aspecto y sanidad de planta y las mejores calificaciones de aspecto y sanidad de mazorca, buena cobertura de mazorca, y los porcentajes más bajos de pudrición de mazorca. Tal es el caso de los híbridos: (CML-492xCML-150)xCLQ-RCWQ50, (CML264Qx CML273Q)xCML491, (CML264QxCML-150)xCML491. Por lo anterior, estos híbridos representan una alternativa para la producción de maíz en el estado de Veracruz (Cuadro 5).

\section{Correlaciones fenotípicas}

De las variables analizadas y utilizando los valores promedio a través de los tres ambientes de evaluación en el experimento de híbridos con alta calidad de proteína, se encontró correlación negativa y altamente significativa entre rendimiento de grano con porcentaje de achaparramiento en planta $(-0,70 * *)$, severidad del daño en planta $(-0,64 * *)$ y significativa con porcentaje de achaparramiento en mazorca $(-0,50 *)$, lo que indica que entre mayor es el daño por achaparramiento en planta y mazorca y severidad, el rendimiento de grano es menor.

La variable porcentaje de achaparramiento en planta registró correlación positiva y altamente significativa con severidad del daño $\left(0,86^{* *}\right)$, con el porcentaje de mazorcas con achaparramiento $\left(0,66^{* *}\right)$ y con sanidad de planta $\left(0,81^{* *}\right)$. Finalmente, se encontró correlación positiva y altamente significativa entre porcentaje de mazorcas con achaparramiento y las variables aspecto de mazorca $\left(0,86^{* *}\right)$ y sanidad de mazorca $\left(0,84^{* *}\right)$. Estos resultados coinciden con lo reportado por Gordon et al. (1997) y Obando et al. (1997), quienes encontraron que la enfermedad del achaparramiento tiene un efecto directo en el rendimiento de grano.

\section{CONCLUSIONES}

En los análisis de varianza combinados para rendimiento, porcentaje de daño por achaparramiento en planta y mazorca y severidad, fue más importante la varianza debida al factor localidades.

Los híbridos de maíz con alta calidad de proteína sobresalientes por su rendimiento y tolerancia a la enfermedad del achaparramiento fueron: (CML264QxCML150)CML491, (CML264Qx CML 
Cuadro 5. Características agronómicas de híbridos de maíz con alta calidad de proteína. Veracruz, México. 2005B.

\begin{tabular}{|c|c|c|c|c|c|c|c|c|c|c|}
\hline Trat. & Genealogía & $\begin{array}{c}\text { Alt. } \\
\text { Pl.(cm) }\end{array}$ & $\begin{array}{c}\text { Alt. } \\
\text { Mz.(cm) }\end{array}$ & $\begin{array}{l}\text { DFM } \\
\text { Cob. }\end{array}$ & API & SPI & AMz & SMz & $\begin{array}{c}\% \\
\text { MzM }\end{array}$ & $\begin{array}{c}\% \\
\text { MzP }\end{array}$ \\
\hline 8 & (CML492xCML-150)xCLQ-RCWQ50 & 246 & 128 & 56 & 1,99 & 2,06 & 1,83 & 2,11 & 1,77 & 5,49 \\
\hline 10 & (CML-264QxCML-273Q)xCML-491 & 231 & 126 & 57 & 2,40 & 2,28 & 1,78 & 1,94 & 1,37 & 2,57 \\
\hline 6 & (CML-264QxCML-150)xCML-491 & 232 & 122 & 56 & 2,32 & 2,33 & 2,17 & 2,22 & 1,98 & 8,38 \\
\hline 13 & (CML264QxCML491)xCLQ-RCWQ10 & 244 & 115 & 55 & 2,34 & 2,67 & 2,11 & 2,33 & 6,40 & 10,7 \\
\hline 7 & (CML144XCML159)xCLQ-RCWQ50 & 236 & 124 & 55 & 2,66 & 2,44 & 2,61 & 2,78 & 2,48 & 10,4 \\
\hline 3 & (CLQ-RCWQ10XCLQ-6315)xCML491 & 243 & 135 & 56 & 2,61 & 2,72 & 2,22 & 2,22 & 2,19 & 11,5 \\
\hline 9 & (CML-492xCML146)xCLQ-RCWQ50 & 242 & 121 & 56 & 2,61 & 2,89 & 2,56 & 2,56 & 1,98 & 6,09 \\
\hline 17 & H-59 RE & 231 & 123 & 54 & 2,62 & 2,55 & 2,33 & 2,45 & 2,35 & 9,48 \\
\hline \multirow[t]{2}{*}{12} & (CLQ-RCWQ10xCLQ-6315)xCML- & & & & & & & & & \\
\hline & 264Q & 231 & 116 & 55 & 2,66 & 2,83 & 2,78 & 2,95 & 10,6 & 12 \\
\hline 18 & H-520 & 242 & 132 & 55 & 2,51 & 2,33 & 2,67 & 2,72 & 2,49 & 6,56 \\
\hline 4 & (CML141xCML142)xCML-491 & 241 & 139 & 56 & 2,78 & 2,67 & 2,78 & 2,67 & 2,00 & 8,68 \\
\hline 5 & (CML264QxCML159) xCML-491 & 233 & 124 & 57 & 2,38 & 2,39 & 2,06 & 2,22 & 2,94 & 4,73 \\
\hline 11 & (CL-RCWQ13xCLQ-6316)xCML-264Q & 237 & 123 & 56 & 2,61 & 2,50 & 2,78 & 2,83 & 11 & 11,2 \\
\hline 14 & (CML264QxCML-491)xCLQ-6315 & 236 & 129 & 57 & 2,56 & 2,78 & 2,83 & 2,89 & 3,37 & 10,2 \\
\hline 2 & (CML492XCML150) xCML-491 & 236 & 122 & 56 & 2,84 & 3,00 & 2,55 & 2,50 & 2,33 & 10,8 \\
\hline 15 & (CML264QxCML491)xCLQ-6316 & 224 & 119 & 57 & 2,44 & 2,78 & 2,56 & 2,61 & 4,75 & 9,95 \\
\hline 16 & (CML144xCML159)xCML-176 RE & 242 & 117 & 56 & 2,61 & 3,17 & 3,11 & 3,22 & 3,52 & 15,2 \\
\hline \multirow[t]{7}{*}{1} & (CML264QxCML-492)xCML-491 & 236 & 120 & 56 & 2,50 & 2,72 & 2,50 & 2,39 & 1,13 & 7,97 \\
\hline & PROMEDIO & 236,76 & 124,12 & 55,88 & 2,52 & 2,62 & 2,46 & 2,13 & 3,59 & 8,99 \\
\hline & $\mathrm{CV}$ (porcentaje) & 5,04 & 9,05 & 1,67 & 13,6 & 13,6 & 15,21 & 13,1 & 33,6 & 33,3 \\
\hline & FSIG & 1 & 1 & 1 & 1 & 1 & 1 & 1 & 1 & 1 \\
\hline & CME & 143 & 126,23 & 0,87 & 0,12 & 0,13 & 0,14 & 0,11 & 0,43 & 0,90 \\
\hline & DMS 0,05 & 11,2 & 10,48 & 0,87 & 0,32 & 0,33 & 0,35 & 0,31 & 0,61 & 0,89 \\
\hline & DMS 0,01 & 14,8 & 13,88 & 1,15 & 0,42 & 0,44 & 0,46 & 0,41 & 0,81 & 1,17 \\
\hline
\end{tabular}

$\mathrm{B}=$ Ciclo primavera verano

1/ Promedio de localidades de Cotaxtla, Tlalixcoyan e Ignacio de la Llave, Ver.

* Significancia de los tratamientos al 0,05 de probabilidad

** Significancia de los tratamientos al 0,01 de probabilidad

Alt. $\mathrm{Pl}=$ Altura de planta; Alt. $\mathrm{Mz}=$ Altura de mazorca; $\mathrm{DFM}=$ Días a floración masculina; APl.= Aspecto de planta;

$\mathrm{SPl}=$ Sanidad de planta; $\mathrm{AMz}=$ Aspecto de mazorca; $\mathrm{SMz}=$ Sanidad de mazorca; \% MzM Cob= Porcentaje de mazorcas con mala cobertura; \% MzP = Porciento de mazorcas podridas.

\section{Q)CML491,CML144xCML159)xCLQ-RCWQ50y CML492xCML150)xCLQRCWQ50}

Las localidades que registraron mayor porcentaje de daño y severidad por "achaparramiento" en planta y mazorca fueron: Ignacio de la Llave, y La Torrecilla, Municipio de Tlalixcoyan, Veracruz, considerándose ambientes aptos para seleccionar genotipos tolerantes a esta enfermedad.

El rendimiento de grano estuvo afectado por el porcentaje de achaparramiento en planta $\left(\mathrm{r}=-0,70^{* *}\right)$, severidad del daño en planta $\left(\mathrm{r}=-0,64^{* *}\right)$ y porcentaje de achaparramiento en mazorca $(\mathrm{r}=-0,50 *)$.

La variable porcentaje de achaparramiento en planta estuvo asociada con severidad del daño $\left(\mathrm{r}=0,86^{* *}\right)$, porcentaje de mazorcas conachaparramiento $\left(\mathrm{r}=0,66^{* *}\right)$ y con sanidad de planta $\left(\mathrm{r}=0,81^{* *}\right)$.

El porcentaje de mazorcas con achaparramiento afectó significativamente las variables aspecto de mazorca $\left(\mathrm{r}=0,86^{* *}\right)$ y sanidad de mazorca $(\mathrm{r}=0,84 * *)$. 


\section{LITERATURA CITADA}

BAJET, N.B.; RENFRO, B.L. 1989. Ocurrence of corn stunt Spiroplasma at different elevations in Mexico. Plant Disease 73: 926-930.

CANO, R.O.; SIERRA, M.; JEFFERS, D.; TOSQUY, O.H.; PALAFOX, C. A.; PRECIADO, R.E. 2000. Respuesta de híbridos de maíz QPM y normales a infestación con Dalbulus maydis vector del achaparramiento del maíz. In: Memorias de la XIII Reunión Científica Tecnológica Veracruz 2000. s/p

DE LEÓN, C. 1984. Enfermedades del maíz. Una guía para su identificación en el campo. 3 ed. El Batán, Texcoco, Edo. de México. Centro Internacional de Mejoramiento de Maíz y Trigo. 114 p.

GARCÍA, E. 1981. Modificaciones al sistema de clasificación climática de Köppen. 3 ed. Universidad Nacional Autónoma de México. Instituto de Geografía. México D.F., México. 252 p.

GORDON, R.; DE GRACIA, N.; FRANCO, J.; GONZÁLEZ, A. 1997. Evaluación de distintas épocas de siembra y la incidencia del achaparramiento en maíz, Azuero, Panamá, 1993-94. In: Síntesis de resultados experimentales del Programa Regional de Maíz (PRM). 1993-1995. CIMMYT-PRM Guatemala. Vol 5: $268-273$.

HENRÍQUEZ, P.; JEFFERS, D. 1997. El achaparramiento del maíz: patógenos, síntomas y diagnostico. In: Síntesis de resultados experimentales del PRM 1993-1995. CIMMYT-PRM Guatemala. Vol 5: 283-290.

IBARRA-APARICIO, G.; MOYA-RAYGOZA, G.; BERLANGA-PADILLA, A. 2005. Efecto de Beauveria bassiana y Metarhizium anisopliae sobre la chicharrita del maíz (Dalbulus maidis) (Delong y Wolcott, 1923) (Hemiptera: Cicadellidae). Folia Entomol. Mex. 44: 1-6.

JEFFERS, D.; MARTÍNEZ-HERNÁNDEZ, L.; BERGVINSON, D.; CÓRDOVA, H.; BECK, D. 2004. Avances en el desarrollo de germoplasma resistente al complejo del achaparramiento del maíz en el CIMMYT. Memorias del XXXI Congreso Nacional de Fitopatología. Resumen C-62.

MERTZ, E. T. 1994. Thirty years of opaque 2 maize. In: Quality Protein Maize. 1964-1994. Proc. of Symp. of Quality Protein Maize. EMBRAPA/CNPMS, Sete Lagoas M. G. Brasil. p 1-10.
MORRIS, M.L.; LÓPEZ, M.A. 2000. Impactos del mejoramiento de maíz en América Latina 1966-1997. México D.F. CIMMYT 45 p.

OBANDO, R.; URBINA, R., MIHM, J.; MENDOZA, M. 1997. Evaluación de materiales con resistencia múltiple a cogollero, barrenadores y achaparramiento. In: Síntesis de resultados experimentales del Programa Regional de Maíz (PRM) 1995-96. Vol. 5: 21-25.

SAGARPA (Secretaría de Agricultura, Ganadería, Desarro1lo Rural, Pesca y Alimentación). 2002. Servicio de Información y Estadística Agroalimentaria y Pesquera. Subsistema de Información Agrícola. México, D.F. (Versión en disco compacto).

SHURTLEFF, C.M. 1980. Compendium of corn diseases. 2 ed. The American Phytopathological Society, St. Paul Minnesota, U.S.A. 105 p.

SIERRA, M. M.; PALAFOX, C. A.; CANO, O.; RODRÍGUEZ, M. F. A.; ESPINOSA, A.; TURRENT, A.; GÓMEZ, N.; CÓRDOVA, H.; VERGARA, N.; AVELDAÑO, S. R.; BARRÓN, S.; ROMERO, J.; CABALLERO, F.; GONZÁLES, C. M.; BETANZOS, E. 2001. Descripción varietal de H-519C, H-553C y V-537C, maíces con alta calidad de proteína para el trópico húmedo de México. INIFAP CIRGOC. Campo Experimental Cotaxtla. Folleto Técnico Núm.30. Veracruz, México. 21 p.

SIERRA, M.; PALAFOX, A.; RODRÍGUEZ, F. A.; ESPINOSA, A.; GÓMEZ, M. N.; CABALLERO, F.; BARRÓN, F. S.; ZAMBADA, M. A. 2004a. H-518 y H-520, híbridos trilineales de maíz para el trópico húmedo de México. INIFAP. CIRGOC. Campo Experimental Cotaxtla. Folleto Técnico Núm. 38. Veracruz, México. 17 p.

SIERRA, M.; BECERRA, E.N.; PALAFOX, A.; JEFFERS, P.D.; CANO, O.; RODRÍGUEZ, F.; TOSQUY, O.H. 2004b. Identificación de híbridos de maíz por su rendimiento y tolerancia a la enfermedad "achaparramiento". Memorias del XXXI Congreso Nacional de Fitopatología. Resumen L-97.

URBINA, R. 1997. Desarrollo de dos poblaciones tropicales de maíz con resistencia al complejo del achaparramiento. In: Síntesis de resultados experimentales del PRM 1993-1995 CIMMYT-PRM Guatemala. Vol 5(1): 15-20.

VASAL, S. K.; VERGARA, A. N.; MC LEAN, S. 1994. Estrategias en el desarrollo de híbridos tropicales de maíz. Agronomía Mesoamericana 5: 184-189.

AGRONOMÍA MESOAMERICANA 18(1): 93-101. 2007 